\title{
Flexible and stretchable electronics for wearable healthcare
}

\author{
Jeroen van den Brand, Margreet de Kok, Ashok \\ Sridhar \\ Holst Centre / TNO \\ High Tech Campus 31 \\ Eindhoven, the Netherlands \\ jeroen.vandenbrand@tno.nl
}

\begin{abstract}
Measuring the quality of human health and wellbeing is one of the key growth areas in our society. Preferably, these measurements are done as unobtrusive as possible. These sensoric devices are then to be integrated directly on the human body as a patch or integrated into garments. This requires the devices to be very thin, flexible and sometimes even stretchable. An overview will be given of recent technology developments in this domain and concrete application examples will be shown.
\end{abstract}

\section{Keywords-flexible electronics, stretchable electronics}

\section{INTRODUCTION}

Today's wearable healthcare tools are complex systems, based on advanced electronics (mixed-signal front-ends, DSP, transceiver, power management, memory ...). Nevertheless, they predominantly rely on rather traditional system design and technology for integration and packaging. These devices are typically built using conventional board-level and/or package-level integration. The result is mostly a system that is not comfortable to wear because it is rigid, large and heavy. In contrast to this, from a user point of view, the device should preferably be comfortable and unnoticeable. Standard integration technologies do typically not fulfil these requirements.

An interesting approach to achieve better wearability is by transforming the flat rigid device into a flexible or stretchable electronics device that can shape around the curved nature of the human body.

The current paper addresses recent technology developments at our institutes that enable this. Two generic complementary platforms will be discussed. The first one is based on organic and large area electronics technologies ('printed electronics') and uses low cost polyester foils. It is specifically targeting low cost, large area devices like health patches. The second one is based on spin-on PI and thin film metallization. This platform targets more advanced wearable and medical devices. The paper also addresses progress in two complementary technologies that are used in both platforms: thin chip integration and a technology for realizing stretchable devices from flexible foils. Finally, concrete application prototypes will be shown in which the platforms and technologies are being exploited.

\author{
Maarten Cauwe, Rik Verplancke, Frederick Bossuyt \\ Johan de Baets, Jan Vanfleteren \\ Imec CMST Technology Park 914, B-9052 \\ Ghent, Belgium
}

\section{TECHNOLOGY ADVANCEMENTS}

\section{A. Organic and large area electronics platform}

Organic and large area electronics (OLAE) uses thin and flexible plastic foils as a base. Mostly polyesters like PET and PEN are chosen because of their low cost and transparent nature. A large part of the electronic functionality is printed on this foil as thin layers of functional ink. The end result of the optimal deployment of OLAE technologies is a very thin and flexible but also low cost electronics device. Because of this nature, OLAE technologies are currently already being industrially employed for healthcare and wearable devices. For example ECG patches but also for glucose diagnostic devices. Up to now however, this primarily concerns low complexity devices. Only coarse pitch circuitry traces are being printed while all more complex functionality (e.g. microcontrollers, radio chips) is integrated into the system on a separate, often rigid, printed circuit board (PCB).

Recent advances in (nano-particle) inks, screens/stencils and process development show promises to realize more complex devices using OLAE technologies. Circuitry patterns with resolutions down to $50 \mu \mathrm{m}$ line/spacing can be achieved. These resolutions are well-in-line with what is needed for typical electronics devices. More complex circuits also involves the ability to realize multilayered circuitry patterns. Also here advances have been made in recent years. Figure 1 shows an example of a 3 layer printed circuit by printing alternating layers of conducting (grey) and dielectric ink (white) on top of each other. Figure 1 also emphasizes one of the clear advantages of OLAE technologies: the base foil has a thickness of only $50 \mu \mathrm{m}$ while the 5 sequentially printed layers only add a maximum of $35 \mu \mathrm{m}$ to the thickness. This results in a very flexible 3 layer PCB having a thickness of less than 150 $\mu \mathrm{m}$.

\section{B. Ultra-thin electronics platform}

More complex, higher end wearable and medical devices often need device (e.g. thinness, flexibility) and circuitry (e.g. pitch, IO count, conductivities) performance that cannot be achieved with OLAE technologies.

To meet these needs, a platform is under development that combines spin-on polyimide layers with thin-film 
metallization. The metallization can be thin $(1 \mu \mathrm{m})$ or thick $(5$ to $10 \mu \mathrm{m})$ copper, but also more biocompatible alternatives using gold or even platinum have been realized.

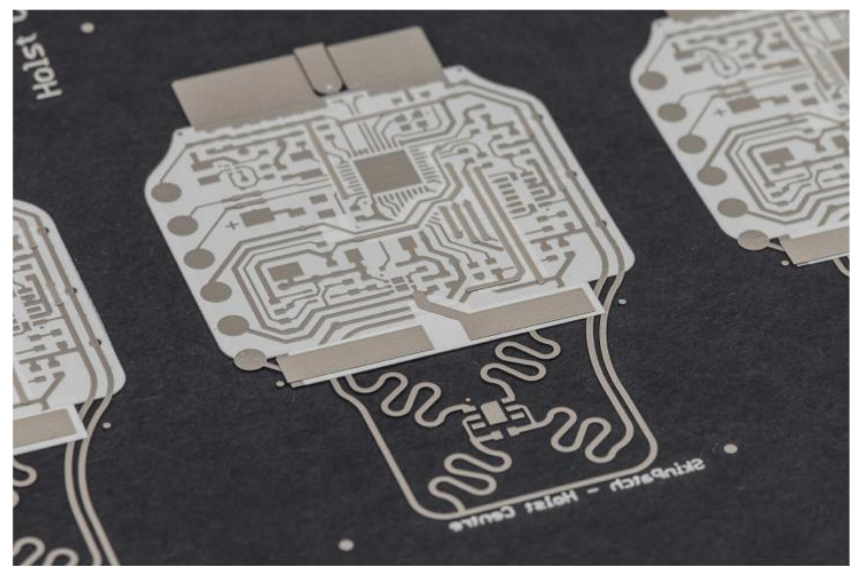

Figure 1. Overview image of a 3 layer printed circuit on a $50 \mu \mathrm{m}$ PET foil. Circuitry is printed with screen printing technology using Ag-filled conductive ink (grey) and dielectric ink (white)

The process starts from a glass carrier substrate, onto which a polyimide layer is spun having a typical thickness of around 5$10 \mu \mathrm{m}$. The ultra-thin chips, having typical thicknesses down to $20 \mu \mathrm{m}$, are subsequently placed in their desired location. A second, photosensitive polyimide layer is spun on top of the chip and consequently patterned to remove the polyimide in the area above the chip. The second polyimide layer thus acts as a planarization layer for the third polyimide layer that carries the interconnection circuitry. This metal layer is realized by vacuum depositing a copper seed layer which is electroplated to the desired thickness. As a final step, the metallization layer is patterned, resulting in a very thin and flexible device having a thickness of about $70 \mu \mathrm{m}$, mounted on a glass carrier. The use of thin film lithographic processes enables to achieve very fine pitches. Currently it is possible to go down to pitches of $65 \mu \mathrm{m}$. Figure 2 shows a typical example. The platform results in very flexible devices: bending radii of less than $4 \mathrm{~mm}$ can be achieved for devices having integrated ultrathin chips.

\section{Thin chip integration}

Most medical and wearable devices need some form of intelligence. For example microcontrollers (to process sensor data) or radio chips (to communicate this data with the outside world).

Integrated circuits are normally supplied in packaged format. Using these bulky and rigid packages has a big impact on the thickness and flexibility advantages of the base foils. At thicknesses below $25 \mu \mathrm{m}$, it is known that silicon becomes flexible [1]. It would thus be preferred to integrate the needed silicon functionality as bare die and in a thinned down form. Several approaches exist to achieve thin silicon chips. Grinding and polishing is one of the more common routes but for example the ChipFilm technology of IMS Chips [1] results in inherently thin silicon chips.

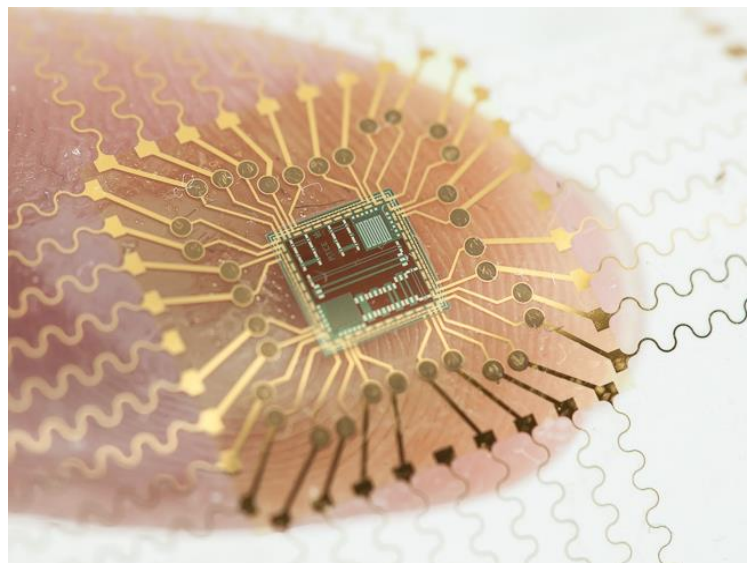

Figure 2. Ultra-thin chip package with fan-out interconnection scheme. The $5 \mathrm{~mm} \times 5 \mathbf{~ m m}$ chip is only $20 \mu \mathrm{m}$ thick and can be bend to a radius of $4 \mathrm{~mm}$.

Considerable work has been performed to better understand the mechanical and functional stability of such thin chips [2] both for the OLAE and the ultra-thin electronics platform. A dedicated four-point mechanical bending test setup has been built in which the bending and electrical behavior of a thin chip can be monitored in-situ. Figure 3 shows some recent results obtained with this setup. Microcontroller chips of different thicknesses were tested for the stability of their internal clock as a function of bending radius. This result shows that not only the mechanical strength of the silicon needs to be taken into account when choosing a chip thickness to achieve a given bending radius, but also the effect of the generated stress on the active layers inside the chip.

The integration of silicon chips with OLAE foils is a challenge because of the low thermal stability of the polyester foils. PEN has a glass transition temperature $(\mathrm{Tg})$ of $\sim 130{ }^{\circ} \mathrm{C}$ and PET of $\sim 85{ }^{\circ} \mathrm{C}$ while poly(imide) has a $\mathrm{Tg}$ of $\sim 350{ }^{\circ} \mathrm{C}$ [3].

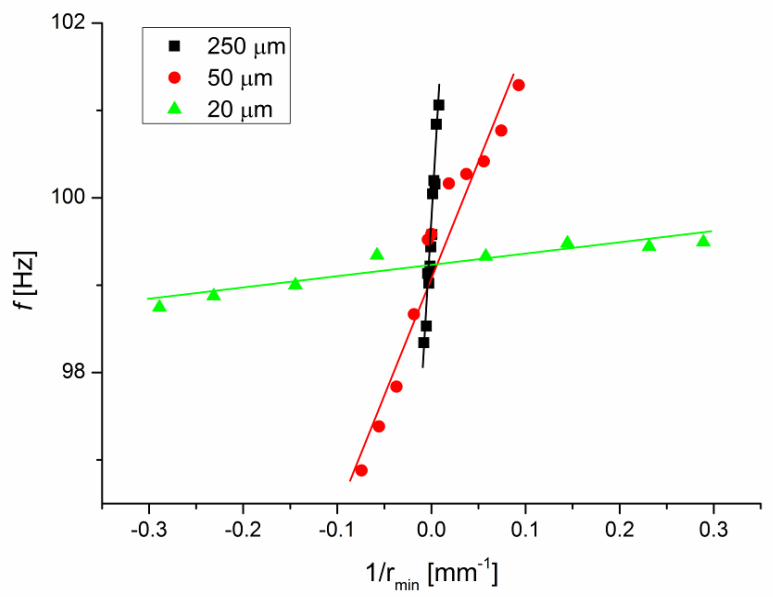

Figure 3: Frequency of the output waveforms of thinned microcontroller dies with three different thicknesses as a function of inverse bending radius. 
This limitation in thermal stability excludes many well established processes for making electronic products, as for example soldering. The integration of silicon chips with OLAE foils is most commonly done using a flip chip process based on conductive adhesives. Specifically work was performed on achieving a reliable and robust chip-foil interconnect with low temperature cure anisotropic conductive adhesive (ACA's) [4, 5]. Figure 4 shows typical reliability results of a $25 \mu \mathrm{m}$ thick silicon chip with a pitch of $250 \mu \mathrm{m}$ attached to a PET foil using an anisotropic conductive adhesive. It can be seen that the low chip thickness results in excellent thermal shock behavior, while the accelerated humidity shows some failure due to hygroscopic swelling of the adhesive. Current activities involve achieving even finer pitches for even thinner chips. Instead of ACA's, high resolution printed isotropic conductive adhesives are being used for OLAE-based applications. In parallel, alternative technologies are being pursued to integrate thin chips $[6,7]$.
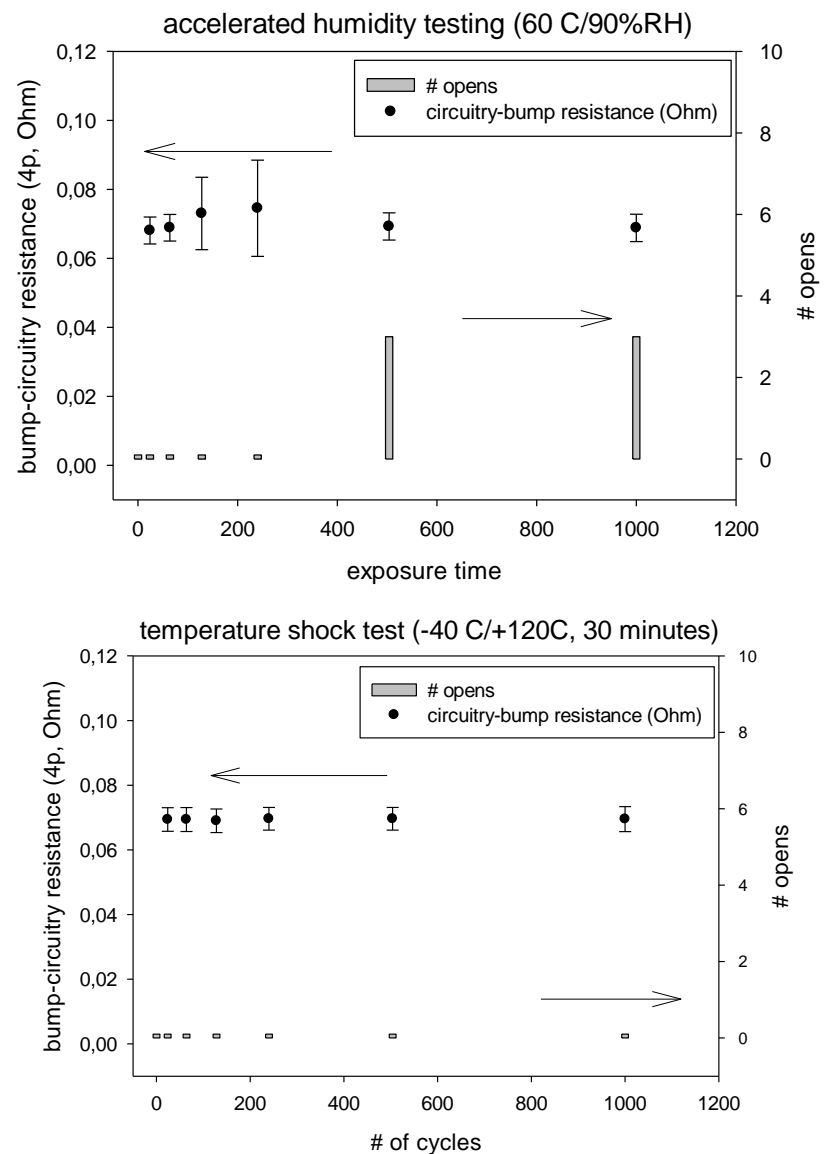

Figure 4. Top: accelerated humidity test results (AHT), Bottom: temperature shock test results (TST)

\section{Stretchable and conformable electronics}

Flexible electronics can meet to a large extent the irregularities of the human body. For increased user comfort, it would however be needed that the electronics device can be deformed in more than one direction simultaneously. The device would then need to be conformable or stretchable.

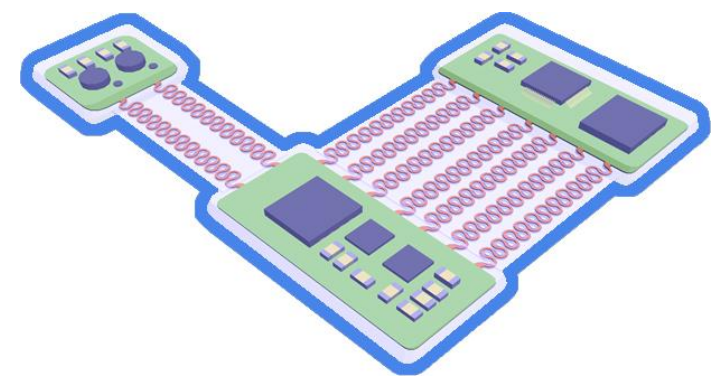

Figure 5. Schematic principle of meander-based stretchable electronics technology.

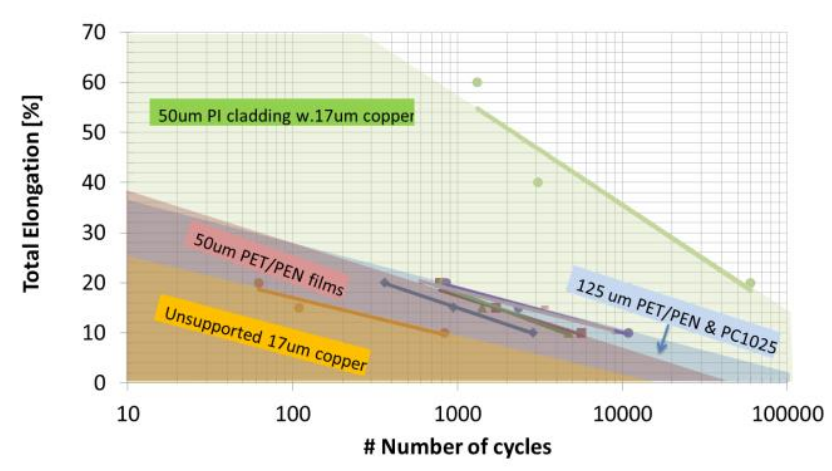

Figure 6. Graph showing the reliability of the meander technology for different substrate types.

In the past years, a technology has been developed which enables the realization of stretchable systems from flexible foils [8]. The basic principle is shown in Figure 5. The electronic functionality is distributed onto electronics islands that are interconnected to each other by meander-shaped interconnects. The whole system is embedded into a stretchable rubber to keep everything together and to protect the device. Upon stretching of the system, the meanders extent by opening up and bending limitedly out-of-plan.

An advantage of this technology over competing stretchable electronics technologies [9] is that it uses standard flex foil manufacturing technologies. The process starts from a normal full area flex foil (polyester, polyimide,...). Circuitry is made with standard printed circuit board technologies (printing, lithography...). Components are also assembled using traditional interconnect technologies (adhesives, soldering). Once finished, the meanders are structured into the foil using for example a laser or die cutting. Finally, the structured foil is embedded inside a rubber material by lamination of a polyurethane or overmoulding with a silicone.

Good stretchability and reliability can be achieved with the technology. Figure 6 shows an overview of typical stabilities for different foil types embedded in a silicone. Unsupported meanders can be stretched almost a thousand times at $10 \%$ elongation. A supporting layer of PET or PEN increases that tenfold, while a $50 \mu \mathrm{m}$ PI cladding achieves over 100.000 cycles at $10 \%$ elongation or more than 1000 cycles at $50 \%$ elongation. 


\section{APLICATION EXAMPLES}

As an illustration of the advancements in the technology, some application example prototypes are shown in Figures 7-9. Figure 7 shows a phototherapy device that is to be worn around the wrist. It contains a matrix of blue LEDs which are considered to relieve pain. The meander-based stretchable electronics technology is applied to ensure the device conforms around the hand. Figure 8 shows an example of a thin $(<150 \mu \mathrm{m})$ multi-purpose sensor. Three circuit layers and a minimum pitch of $100 \mu \mathrm{m}(50 \mu \mathrm{m}$ line/spacing) were successfully and reproducibly realized using printing on a polyester foil. Ultrathin chips (microcontroller, radio chip, 25 $\mu \mathrm{m}$ thickness) were successfully integrated. Figure 9 shows an example of a more complex medical device, realized using the ultrathin electronics platform. It consists of a 4 x 4 microelectrode array (shown in the inset), which is routed towards a $30 \mu \mathrm{m}$ thick custom designed ASIC. The ASIC enables multi-channel brain recording and stimulation. Its compact, lightweight and conformable nature makes the system ideally suited for use with small rodents, such as rats.
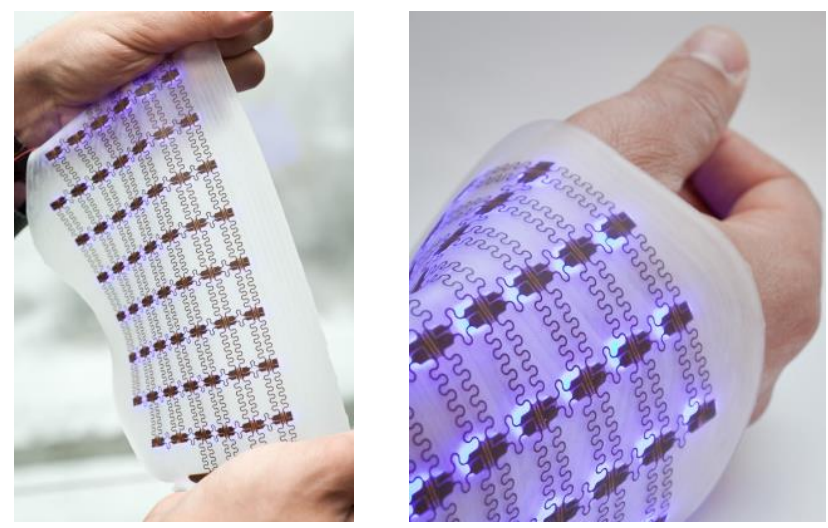

Figure 7. Phototherapy device realized using meanderbased stretchable electronics technology. Work performed in the framework of the FP7 EU Project PlaceIT.

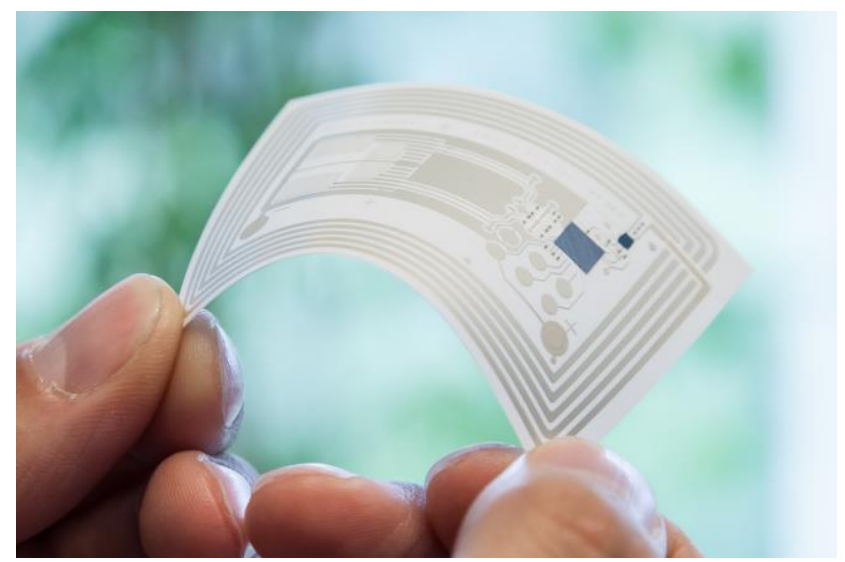

Figure 8. Flexible sensor patch based on OLAE technologies (printing of circuitry) with ultrathin (25 $\mu \mathrm{m}$ thick) silicon chips.

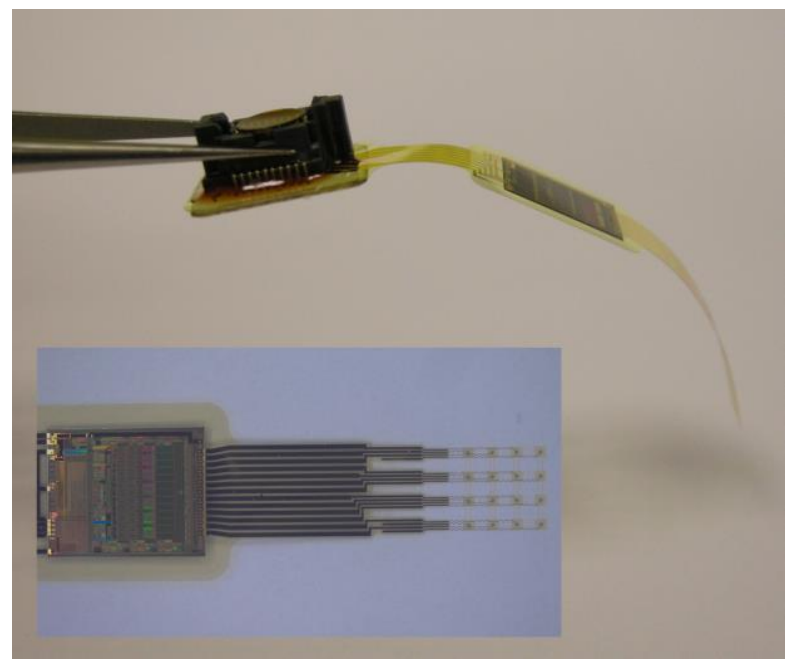

Figure 9. Lightweight, conformable system consisting of a $4 \times 4$ microelectrode array and ASIC for multi-channel brain recording and stimulation.

[1] J. N. Burghartz, W. Appel, C. Harendt, H. Rempp, H. Richter, and M. Zimmermann, "Ultra-thin chip technology and applications, a new paradigm in silicon technology," Solid-State Electronics, vol. 54, pp. 818-829, 2010.

[2] D. van den Ende, F. Verhoeven, P. van der Eijnden, R. Kusters, A. Sridhar, M. Cauwe, et al., "High curvature bending characterization of ultra-thin chips and chip-on-foil assemblies," in 2013 European Microelectronics Packaging Conference, ed New York: Ieee, 2013.

[3] J. Fjelstad, Flexible Circuit Technology, 3rd ed.: BR Publishing Inc, 2007.

[4] J. v. d. Brand, E. Veninga, R. Kusters, T. Podprocky, and A. Dietzel, "Flipchip Bonding of Si Chip on Flexible PEN Foil using Novel Electronic $100 \mu \mathrm{m}$ Pitch Fan-out Circuitry," Matererial Research Society Symposium Proceedings, vol. 1030, pp. G05-10-17, 2008.

[5] J. v. d. Brand, R. Kusters, M. Heeren, B. v. Remoortere, and A. Dietzel, "Flipchip bonding of ultrahin Si dies onto PEN/PET substrates with low cost circuitry " presented at the ESTC 2010, Berlin, 2010.

[6] M. Cauwe, B. Vandecasteele, J. De Baets, J. van den Brand, R. Kusters, A. Sridhar, et al., "A chip embedding solution based on low-cost plastic materials as enabling technology for smart labels," 2012 4th Electronic SystemIntegration Technology Conference (Estc), p. 6, 2012.

[7] A. Sridhar, M. Cauwe, H. Fledderus, R. H. L. Kusters, J. van den Brand, and Ieee, "Novel Interconnect

Methodologies for Ultra-thin Chips on Foils," 2012 Ieee 62nd Electronic Components and Technology Conference (Ectc), pp. 238-244, 2012.

[8] F. Bossuyt, T. Vervust, and J. Vanfleteren, "Stretchable Electronics Technology for Large Area Applications: Fabrication and Mechanical Characterization," Ieee Transactions on Components Packaging and Manufacturing Technology, vol. 3, pp. 229-235, Feb 2013. J. A. Rogers, T. Someya, and Y. Huang, "Materials and mechanics for stretchable electronics," Science, vol. 327, pp. 1603-7, Mar 262010. 